\title{
Compositional Circular Assume-Guarantee Rules Cannot Be Sound and Complete
}

\author{
Patrick Maier \\ Max-Planck-Institut für Informatik \\ Stuhlsatzenhausweg 85, 66123 Saarbrücken, Germany \\ maier@mpi-sb.mpg.de
}

\begin{abstract}
Circular assume-guarantee reasoning is used for the compositional verification of concurrent systems. Its soundness has been studied in depth, perhaps because circularity makes it anything but obvious. In this paper, we investigate completeness. We show that compositional circular assume-guarantee rules cannot be both sound and complete.
\end{abstract}

\section{Introduction}

The goal in compositional verification of concurrent systems is to prove that a complex system, a parallel composition of several subsystems, satisfies a complex property, a conjunction of several simpler properties. In principle, verification tools can attack such a goal directly, at least if the complex system is finite still. However, the state space of the system may be exponentially larger than that of any subsystem - a phenomenon called state explosion — which may cause verification to become intractable in practice. Compositional verification tries to use the modular structure of complex systems and properties to decompose intractable verification tasks into a bunch of smaller, hopefully tractable subtasks; ideally, each subtask only establishes properties of a single subsystem in isolation. Later, one deduces from all these subtasks via a suitable proof rule that the original complex system satisfies the desired complex property.

Systems, Properties. We model systems and properties uniformly as elements of $\mathbf{S}=\langle S, \wedge, 1, \leq\rangle$, a meet-semilattice with one, i. e., a partial order $\langle S, \leq\rangle$ with greatest element 1 in which the greatest lower bound $x \wedge y$ of any two elements $x$ and $y$ exists. In this model, the expression $x \leq y$ can have three different readings depending on whether $x$ and $y$ denote systems or properties, respectively. If both are systems then $x \leq y$ means that $x$ refines $y$, if both are properties then it means that $x$ entails $y$, and if $x$ is a system and $y$ a property then $x \leq y$ expresses that $x$ satisfies $y$. Likewise, $x \wedge y$ denotes composition if $x$ and $y$ are systems, it denotes conjunction if $x$ and $y$ are properties, and if $x$ is system and $y$ a property then $x \wedge y-x$ constrained by $y$ - is the coarsest refinement of $x$ that satisfies $y$. Thus, all we require of systems (properties) is that refinement (entailment) is an order and that composition (conjunction) is an associative commutative and idempotent operation which respects the order. Section 4 will show that this 
abstract algebraic setting already suffices for proving incompleteness of circular assume-guarantee reasoning. In particular, no notion of computation is required, unlike in the proofs for soundness.

Example 1. Meet-semilattices are a natural model for systems and properties. For instance, in (linear-time) temporal verification, often one views systems and properties as languages over some non-empty (and possibly infinite) alphabet $\Sigma$. In this setting, refinement and entailment correspond to language inclusion, and composition and conjunction correspond to language intersection, so we have a meet-semilattice structure. How that meet-semilattice actually looks like, depends on the type of properties we want to verify.

By the characterization in [3], safety properties are expressible as prefixclosed $*$-languages. I. e., a safety property may be viewed as a subset $L$ of $\Sigma^{*}$ such that for all $w \in \Sigma^{*}$, if $w$ belongs to $L$ then all prefixes of $w$ belong to $L$, too. So for verification of safety properties, the meet-semilattice is the set of prefix-closed $*$-languages (over $\Sigma$ ). Note that these are exactly the languages generated by (possibly infinite) labeled state transition graphs, which are a natural representation of systems. In general, when verifying arbitrary temporal properties, the meet-semilattice will be the set of $\omega$-languages (over $\Sigma$ ), i. e., the power set of $\Sigma^{\omega}$. Here, natural representations of systems and properties are some more elaborate variants of state transition graphs, for instance fair transition systems [14] or (possibly infinite state) $\omega$-automata [19].

Proof Rules. In general, there are two kinds of proof rules for compositional verification, non-circular and circular ones. We show some examples to demonstrate the difference. Let $s_{1}, s_{2} \in S$ be systems and $p_{1}, p_{2} \in S$ properties and suppose we want to verify that the composition of $s_{1}$ and $s_{2}$ satisfies the conjunction of $p_{1}$ and $p_{2}$. (1) shows two non-circular rules for this purpose. Both rules decompose the goal into two subgoals, where the subgoals of the first rule state that system $s_{i}$ satisfies property $p_{i}$, or in other words: $s_{i}$ guarantees $p_{i}$. The second rule differs only in the second subgoal, which states that $s_{2}$ constrained by $p_{1}$ satisfies $p_{2}$, or in other words: if $p_{1}$ is assumed then $s_{2}$ guarantees $p_{2}$ hence the wide-spread term assume-guarantee rule.

$$
\frac{s_{1} \leq p_{1} \quad s_{2} \leq p_{2}}{s_{1} \wedge s_{2} \leq p_{1} \wedge p_{2}} \quad \frac{s_{1} \leq p_{1} \quad p_{1} \wedge s_{2} \leq p_{2}}{s_{1} \wedge s_{2} \leq p_{1} \wedge p_{2}}
$$

Going one step further and also introducing an assumption in the first subgoal, we obtain the circular rule (2).

$$
\frac{p_{2} \wedge s_{1} \leq p_{1} \quad p_{1} \wedge s_{2} \leq p_{2}}{s_{1} \wedge s_{2} \leq p_{1} \wedge p_{2}}
$$

Unlike the non-circular rules, (2) is unsound; for instance, if both systems are 1 , the greatest element in $\mathbf{S}$, and both properties are equal and different from 1 then both premises hold but the conclusion does not. As soundness is indispensable, rule (2) must be restricted by a side condition which excludes cases as 
the one above. Such circularity-breaking side conditions do exist; in fact, quite a number of restricted variants of (2) are proven sound (by induction usually) in the literature, see 14,111720 to name just a few.

Completeness. As there are many variants of sound circular assume-guarantee rules, the question arises whether some are better than others. An important criterion for rating rules is the restrictiveness of the side condition; if the side condition is overly restrictive the rule is applicable to few cases only, hence it is considered worse than a variant with a less restrictive side condition (as long as that variant is sound still). In the best case, the rule is complete, i. e., the side condition is true whenever premises and conclusion are true. Thus, the side condition of a complete rule does not restrict the rule unnecessarily since it is true whenever the rule should be applicable. Note however, that the side condition is not redundant in complete rules; it may still be indispensable for proving soundness.

Compositionality. Recall that compositional verification seeks to reduce a large, intractable goal into many smaller subgoals. The rules in (11) and (2) support this approach as the premises of these rules are less complex than their conclusions. In particular, no premise involves the composition of the systems $s_{1}$ and $s_{2}$ any more, so verification of the subgoals is more likely to be tractable than direct verification of the goal. However, when using a circular rule which is restricted by a side condition, it does not suffice to verify the subgoals that arise from the premises; additionally, we need to prove that the side condition holds. It may be the case that this proof requires to consider both systems simultaneously - e.g., for establishing some mutual exclusion property — and thus involves some aspects of the composition, which is against the spirit of compositional verification. Therefore, a rule can be called compositional only if checking the side condition is possible without taking into account both systems simultaneously, i. e., only if the side condition is expressible as a boolean combination of subconditions, each of which involves at most one of the systems.

Plan. Section 2 formally presents proof rules which are restricted by a side condition and defines soundness and completeness. Section 3 specifies what we mean by circular assume-guarantee reasoning in the context of compositional verification and formalizes the precise requirements for rules to be compositional. Section 4 proves the main result that compositional circular assume-guarantee rules cannot be both sound and complete. Finally, Section [5] discusses related work and Section $[6$ concludes. Proofs which have been omitted here due to lack of space can be found in [13].

\section{Inference Rules}

Terms, Formulas. We fix a set of variables Var. Terms are built inductively from variables in Var, the nullary operator $\top$, called top, and the binary operator 
$\sqcap$, called meet. We consider top neutral w.r.t. meet, which is seen as associative, commutative and idempotent. We call a term $t$ atomic iff $t$ is a variable or $t$ is top. By var $(t)$, we denote the set of variables occuring in $t$, and we say that a term $t^{\prime}$ is a subterm of $t$ iff $\operatorname{var}\left(t^{\prime}\right) \subseteq \operatorname{var}(t)$.

A formula $\varphi$ is a pair $\left\langle t, t^{\prime}\right\rangle$ of terms, written as $t \sqsubseteq t^{\prime}$. We refer to $t$ as the left-, to $t^{\prime}$ as the right-hand side of $\varphi$. We denote the set of variables occuring in $\varphi$ by $\operatorname{var}(\varphi)$, i. e., $\operatorname{var}(\varphi)=\operatorname{var}(t) \cup \operatorname{var}\left(t^{\prime}\right)$, and for every set of formulas $\Phi$, we define $\operatorname{var}(\Phi)=\bigcup_{\varphi \in \Phi} \operatorname{var}(\varphi)$.

Truth, Entailment. We fix $\mathbf{S}=\langle S, \wedge, 1, \leq\rangle$, a non-trivial meet-semilattice with one. By $\mathrm{Val}$, we denote the set of valuations, i. e., the set of total functions from $\operatorname{Var}$ to $S$. We extend a valuation $\alpha$ to terms in the canonical way, i.e., $\alpha(\top)=1$ and $\alpha\left(t_{1} \sqcap t_{2}\right)=\alpha\left(t_{1}\right) \wedge \alpha\left(t_{2}\right)$. Note that we may view any term $t$ as a total function from Val to $S$ by defining the function application $t(\alpha)$ as $\alpha(t)$.

We say that a formula $t \sqsubseteq t^{\prime}$ is true under a valuation $\alpha$, denoted by $\alpha \models$ $t \sqsubseteq t^{\prime}$, iff $\alpha(t) \leq \alpha\left(t^{\prime}\right)$. We extend truth to sets of formulas, i. e., $\alpha \models \Phi$ iff $\alpha \models \varphi$ for all $\varphi \in \Phi$.

We say that $\Phi$ entails $\Psi$, denoted by $\Phi \models \Psi$, iff for all $\alpha \in V a l, \alpha \models \Phi$ implies $\alpha \models \Psi$. We say that $\Phi$ is equivalent to $\Psi$, denoted by $\Phi \equiv \Psi$, iff $\Phi \models \Psi$ and $\Psi \models \Phi$. Note that in sets of formulas the operators top and meet are redundant on right-hand sides since for terms $t, t_{1}^{\prime}, t_{2}^{\prime}$, we have the equivalences $\{t \sqsubseteq \top\} \equiv \emptyset$ and $\left\{t \sqsubseteq t_{1}^{\prime} \sqcap t_{2}^{\prime}\right\} \equiv\left\{t \sqsubseteq t_{1}^{\prime}, t \sqsubseteq t_{2}^{\prime}\right\}$.

Relations. Let $X, Y$ and $Z$ be sets and $n \in \mathbb{N}$. Given $n$ functions $f_{1}, \ldots, f_{n}$ : $X \rightarrow Y$ and an $n$-ary function $g: Y^{n} \rightarrow Z$, we define the $n$-ary composition of $g$ and $f_{1}, \ldots, f_{n}$ as the function $g\left[f_{1}, \ldots, f_{n}\right]: X \rightarrow Z$ such that for all $x \in X$, $g\left[f_{1}, \ldots, f_{n}\right](x)=g\left(f_{1}(x), \ldots, f_{n}(x)\right)$.

Let $n \in \mathbb{N}$, let $t_{1}, \ldots, t_{n}$ be $n$ terms and let $C: S^{n} \rightarrow\{0,1\}$, i. e., $C$ is the characteristic function of an $n$-ary relation on $S$, the carrier of our fixed meet-semilattice $\mathbf{S}$. Viewing terms as functions from $\mathrm{Val}$ to $S$, the function $C\left[t_{1}, \ldots, t_{n}\right]: \operatorname{Val} \rightarrow\{0,1\}$ is well-defined - it is the characteristic function of some set of valuations - and we say that $C$ is associated with the terms $t_{1}, \ldots, t_{n}$. Note that for every enumeration $x_{1}, \ldots, x_{m}$ of a superset of the variables occuring in the terms $t_{1}, \ldots, t_{n}$ there is a unique function $C^{\prime}: S^{m} \rightarrow\{0,1\}$ such that $C^{\prime}\left[x_{1}, \ldots, x_{m}\right]=C\left[t_{1}, \ldots, t_{n}\right]$. Therefore, without loss of generality, we may assume that the associated terms are variables.

A relation $\Gamma$ is an $n$-ary function $C: S^{n} \rightarrow\{0,1\}$ associated with $n$ variables $x_{1}, \ldots, x_{n}$, i. e., $\Gamma=C\left[x_{1}, \ldots, x_{n}\right]$. We denote the set of variables occuring in $\Gamma$ by $\operatorname{var}(\Gamma)$, i. e., $\operatorname{var}(\Gamma)=\left\{x_{1}, \ldots, x_{n}\right\}$.

We define a notion of truth for relations, similar to the one for formulas. We say that a relation $C\left[x_{1}, \ldots, x_{n}\right]$ is true under a valuation $\alpha$, denoted by $\alpha \models C\left[x_{1}, \ldots, x_{n}\right]$, iff $C\left[x_{1}, \ldots, x_{n}\right](\alpha)=1$. Rewriting this with the definition of $n$-ary composition, we see that $\alpha \models C\left[x_{1}, \ldots, x_{n}\right]$ iff $C\left(\alpha\left(x_{1}\right), \ldots, \alpha\left(x_{n}\right)\right)=1$. We say that a relation $\Gamma$ is true iff $\alpha=\Gamma$ for all $\alpha \in V a l$. Note that every set of formulas $\Phi$ may be expressed by an equivalent relation $\Gamma_{\Phi}$ with $\operatorname{var}\left(\Gamma_{\Phi}\right)=\operatorname{var}(\Phi)$, where for all valuations $\alpha, \alpha \models \Phi$ iff $\alpha \models \Gamma_{\Phi}$. However, relations are strictly more 
expressive than formulas. For instance, inequality of two distinct variables $x$ and $y$ is not expressible by formulas, i. e., there is no set of formulas $\Phi$ such that for all $\alpha \in \operatorname{Val}, \alpha=\Phi$ iff $\alpha(x) \neq \alpha(y)$.

Inference Rules. An inference rule (or rule, for short) $R$ is a triple $\langle\Phi, \psi, \Gamma\rangle$, where the premises $\Phi$ are a finite set of formulas, the conclusion $\psi$ is a formula, and the side condition $\Gamma$ is a relation. We say that $R$ is syntactic iff the side condition $\Gamma$ is true. We write a rule $R$ as $R: \Phi / \psi$ if $\Gamma$ or

$$
R: \frac{\varphi_{1} \ldots \varphi_{m}}{\psi} \text { if } C\left[x_{1}, \ldots, x_{n}\right]
$$

when $\Phi=\left\{\varphi_{1}, \ldots, \varphi_{m}\right\}$ and $\Gamma=C\left[x_{1}, \ldots, x_{n}\right]$. If $R$ is syntactic then we may omit the side condition and write $R: \Phi / \psi$, simply. Without loss of generality we will assume that the right-hand sides of all premises are atomic and that $\operatorname{var}(\psi) \cup \operatorname{var}(\Gamma) \subseteq \operatorname{var}(\Phi)$, i. e., every variable of the conclusion or the side condition occurs in the premises.

Soundness, Completeness. Let $R: \Phi / \psi$ if $\Gamma$ be an inference rule. We say that $R$ is sound iff for all valuations $\alpha, \alpha \models \Phi$ and $\alpha \models \Gamma$ implies $\alpha \models \psi$. We say that $R$ is syntactically sound iff $\Phi \models \psi$. Note that syntactical soundness implies soundness, and every sound syntactic rule is syntactically sound.

We say that $R$ is complete iff for all valuations $\alpha, \alpha=\Phi$ and $\alpha=\psi$ implies $\alpha \models \Gamma$. Note that every syntactic rule is complete, trivially. Also note that for syntactically sound rules completeness is not an issue, as every syntactically sound rule $R: \Phi / \psi$ if $\Gamma$ can be transformed by omitting the side condition into the (sound and complete) syntactic rule $R^{\prime}: \Phi / \psi$. Hence, there is no reason why a syntactically sound rule should be restricted by a side condition.

Example 2. Assume that $\mathbf{S}=\langle S, \wedge, 1, \leq\rangle$ is the four-element meet-semilattice which is not a chain. Let $s_{1}, s_{2}, p_{1}$ and $p_{2}$ be four distinct variables, where we think of the $s_{i}$ as representing systems and of the $p_{j}$ as representing properties. We define the rules $R_{1}$ and $R_{2}$, where

$$
R_{k}: \frac{p_{2} \sqcap s_{1} \sqsubseteq p_{1} \quad p_{1} \sqcap s_{2} \sqsubseteq p_{2}}{s_{1} \sqcap s_{2} \sqsubseteq p_{1} \sqcap p_{2}} \text { if } C_{k}\left[s_{1}, s_{2}, p_{1}, p_{2}\right]
$$

and for all $a, b, c, d \in S, C_{1}(a, b, c, d)=1$ iff $c$ and $d$ are incomparable, and $C_{2}(a, b, c, d)=1$ iff $a \wedge b \leq c$ and $a \wedge b \leq d$. Both rules are sound as both side conditions are restrictive enough to prevent unsound circular reasoning. $R_{2}$ is trivially complete as $C_{2}\left[s_{1}, s_{2}, p_{1}, p_{2}\right]$ is equivalent to the conclusion. However, $R_{1}$ is incomplete as, for instance, it is not applicable to the (trivial) case when both properties equal 1 . Note that the relation $C_{1}\left[s_{1}, s_{2}, p_{1}, p_{2}\right]$ is not expressible by formulas, which demonstrates that the language of side conditions is more expressive than languages of premises and conclusions. 


$$
\begin{array}{ll}
R_{3}: \frac{s_{1} \sqsubseteq p_{1} s_{2} \sqsubseteq p_{2}}{s_{1} \sqcap s_{2} \sqsubseteq p_{1} \sqcap p_{2}} & R_{6}: \frac{p_{2} \sqcap s_{1} \sqsubseteq p_{1} \quad p_{1} \sqcap s_{2} \sqsubseteq p_{2}}{s_{1} \sqcap s_{2} \sqsubseteq p_{1} \sqcap p_{2}} \\
R_{4}: \frac{s_{1} \sqsubseteq p_{1} \quad p_{1} \sqcap s_{2} \sqsubseteq p_{2}}{s_{1} \sqcap s_{2} \sqsubseteq p_{1} \sqcap p_{2}} & R_{7}: \frac{p_{3} \sqcap s_{1} \sqsubseteq p_{1} \quad p_{1} \sqcap s_{2} \sqsubseteq p_{2}}{s_{1} \sqcap s_{2} \sqsubseteq p_{1} \sqcap p_{2}} \\
R_{5}: \frac{p_{3} \sqcap s_{1} \sqsubseteq p_{1} \quad p_{1} \sqcap s_{2} \sqsubseteq p_{2}}{p_{3} \sqcap s_{1} \sqcap s_{2} \sqsubseteq p_{1} \sqcap p_{2}} & R_{8}: \frac{p_{1} \sqcap s_{1} \sqsubseteq \top \quad p_{2} \sqsubseteq s_{2}}{p_{1} \sqcap s_{1} \sqsubseteq s_{2} \sqcap p_{2}}
\end{array}
$$

Fig. 1. Sample assume-guarantee rules

\section{Assume-Guarantee Rules}

Assume-Guarantee Rules. We call an inference rule $R: \Phi / \psi$ if $\Gamma$ an assumeguarantee rule (or A-G rule, for short) iff for all premises $\varphi \in \Phi$, the left-hand side of $\psi$ and the right-hand side of $\varphi$ do not share any variables. We call an A-G rule $R: \Phi / \psi$ if $\Gamma$ circular iff $\Phi \not \models \psi$.

Example 3. As the definition of A-G rules does not involve the side condition, we may illustrate it using syntactic rules only, see figure 1 There, $s_{1}, s_{2}, p_{1}, p_{2}$ and $p_{3}$ are five distinct variables, where the system/property distinction is as in example 2

The rules $R_{3}, R_{4}$ and $R_{5}$ are non-circular A-G rules. Note the second premise of $R_{4}$, which may be read as assuming the property $p_{1}$ the system $s_{2}$ guarantees the property $p_{2}$. Likewise, the conclusion of $R_{5}$ may be read as assuming $p_{3}$ the composition of $s_{1}$ and $s_{2}$ guarantees both $p_{1}$ and $p_{2}$. This should explain where the term assume-guarantee rule comes from.

The rules $R_{6}, R_{7}$ and $R_{8}$ (and also $R_{1}$ and $R_{2}$ from example 2) are circular A-G rules as they are not syntactically sound. The term circular is justified for $R_{6}$, whose premises express circular assume-guarantee dependencies between the properties $p_{1}$ and $p_{2}$. For $R_{7}$ and $R_{8}$, however, there is no circularity in the premises. In the case of $R_{7}$, unsoundness arises from the unresolved assumption $p_{3}$; compare to $R_{5}$ where that assumption is resolved. $R_{8}$ is unsound because it is nonsense, it serves to demonstrate that not every assume-guarantee rule has a meaningful reading. So, the term circular should not be taken literally, rather it is an abstraction capturing the most important property of circular assumeguarantee reasoning, namely its lack of syntactical soundness.

The following propositions provide an alternative characterization of circularity resp. a sufficient criterion for the truth of the premises of an A-G rule.

Proposition 1. An $A-G$ rule $R: \Phi / t_{\psi} \sqsubseteq t_{\psi}{ }^{\prime}$ if $\Gamma$ is circular if and only if $\Phi \not \forall t_{\psi} \sqsubseteq x$ for some $x \in \operatorname{var}\left(t_{\psi}{ }^{\prime}\right)$.

Proposition 2. Let $R: \Phi / t_{\psi} \sqsubseteq t_{\psi}^{\prime}$ if $\Gamma$ be an $A-G$ rule and let $\alpha$ be a valuation. If for all $x, y \in \operatorname{var}(\Phi) \backslash \operatorname{var}\left(t_{\psi}\right)$, 
$-\Phi \models t_{\psi} \sqsubseteq x$ implies $\alpha(x)=1$, and

- $\Phi \not \forall t_{\psi} \sqsubseteq x$ and $\Phi \not t_{\psi} \sqsubseteq y$ implies $\alpha(x)=\alpha(y)$,

then $\alpha=\Phi$.

A-G Rules for Compositional Verification. As has already been hinted in example 3, for the purpose of verification we distinguish systems and properties, so we partition our variable set Var into system variables $s_{i}$ and property variables $p_{j}$. Section 4 will show that already the composition of only two systems exhibits the incompleteness of compositional circular assume-guarantee reasoning, so actually we can restrict the variable set to $\operatorname{Var}=\left\{s_{1}, s_{2}\right\} \uplus\left\{p_{1}, p_{2}, p_{3}, \ldots\right\}$.

The goal of compositional verification is to establish that the composition of some systems (in our case, $s_{1}$ and $s_{2}$ ) guarantees some property (possibly assuming some other property). So for an A-G rule to be useful for compositional verification, $s_{1} \sqcap s_{2}$ must be a subterm of the left-hand side of the conclusion, which we will implicitly assume henceforth. Thus, without loss of generality we may assume that an A-G rule $R$ is presented in the form

$$
R: \frac{\varphi_{1} \ldots \varphi_{m}}{t_{\psi} \sqsubseteq t_{\psi}^{\prime}} \text { if } C\left[s_{1}, s_{2}, p_{1}, \ldots, p_{n}\right]
$$

where $\left\{s_{1}, s_{2}\right\} \subseteq \operatorname{var}\left(t_{\psi}\right)$ and $\operatorname{var}\left(\left\{\varphi_{1}, \ldots, \varphi_{m}, t_{\psi} \sqsubseteq t_{\psi}{ }^{\prime}\right\}\right)=\left\{s_{1}, s_{2}, p_{1}, \ldots, p_{n}\right\}$. The latter requirement can always be achieved by renaming some property variables and extending and reordering the associated variables in the side condition. By the definition of A-G rules, $\operatorname{var}\left(t_{\psi}\right) \cap \operatorname{var}\left(t^{\prime}\right)=\emptyset$ for every premise $t \sqsubseteq t^{\prime}$, so $t^{\prime} \in\left\{\top, p_{1}, \ldots, p_{n}\right\}$ as we assume the right-hand sides of premises to be atomic.

Compositionality. Let $R: \Phi / \psi$ if $C\left[s_{1}, s_{2}, p_{1}, \ldots, p_{n}\right]$ be an A-G rule. We will call $R$ compositional if it avoids the system composition $s_{1} \sqcap s_{2}$ in the premises as well as in the side condition. Formally, we say that $R$ is compositional in the premises iff $s_{1} \sqcap s_{2}$ is not a subterm of any left-hand side in $\Phi$. We say that $R$ is compositional in the side condition iff $C\left[s_{1}, s_{2}, p_{1}, \ldots, p_{n}\right]$ is expressible as a boolean combination of relations whose associated variables either do not include $s_{1}$ or $s_{2}$. I. e., $R$ is compositional in the side condition iff there are $r_{1}, r_{2} \in \mathbb{N}$, a $\left(r_{1}+r_{2}\right)$-ary boolean function $F:\{0,1\}^{r_{1}+r_{2}} \rightarrow\{0,1\}$ and $r_{1}+r_{2}(n+1)$-ary functions $C_{1}^{1}, \ldots, C_{1}^{r_{1}}, C_{2}^{1}, \ldots, C_{2}^{r_{2}}: S^{n+1} \rightarrow\{0,1\}$ such that

$$
C\left[s_{1}, s_{2}, \tilde{p}\right]=F\left[C_{1}^{1}\left[s_{1}, \tilde{p}\right], \ldots, C_{1}^{r_{1}}\left[s_{1}, \tilde{p}\right], C_{2}^{1}\left[s_{2}, \tilde{p}\right], \ldots, C_{2}^{r_{2}}\left[s_{2}, \tilde{p}\right]\right]
$$

where $\tilde{p}$ abbreviates the enumeration $p_{1}, \ldots, p_{n}$. We say that $R$ is compositional iff it is compositional in the premises and in the side condition.

One may think of the above functions $C_{i}^{k}$ as abstracting the system $s_{i}$ together with the properties $p_{1}, \ldots, p_{n}$ to a boolean value. Actually, we can relax the above definition of compositionality in the side condition from boolean to arbitrary finitary abstractions $C_{i}^{k}$. I. e., $R$ is compositional in the side condition iff there are a finite set $D$ and $r_{1}, r_{2} \in \mathbb{N}$ and $F: D^{r_{1}+r_{2}} \rightarrow\{0,1\}$ and $C_{1}^{1}, \ldots, C_{1}^{r_{1}}, C_{2}^{1}, \ldots, C_{2}^{r_{2}}: S^{n+1} \rightarrow D$ such that the equation (3) holds. 
0

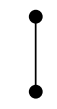

1

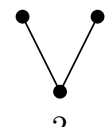

2

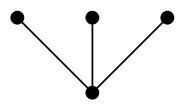

3

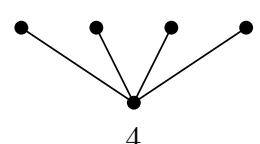

Fig. 2. Forks of width 0 to 4

Example 4. Recall the A-G rules $R_{1}$ to $R_{8}$ from the examples 2]and 3 All these rules are compositional in the premises, and the syntactic rules $R_{3}$ to $R_{8}$ are compositional in the side condition, trivially. The rule $R_{1}$ is also compositional in the side condition but $R_{2}$ is not.

\section{Incompleteness of Compositional Rules}

Forks. We say that $Y \subseteq S$ is a fork iff there is $x \in Y$ such that for all $y, z \in Y$, $y \neq z$ implies $x=y \wedge z$; if $Y$ is infinite then we say that $Y$ is a fork of infinite width, otherwise the size of $Y$ is $m \in \mathbb{N}$ and we say that $Y$ is a fork of width $m-1$. Note that if $\mathbf{S}$ contains a fork of infinite width then it also contains forks of width $m$ for every $m \in \mathbb{N}$.

Example 5. Some forks of finite width are depicted in figure2, Note that if $\mathbf{S}$ is a chain then it contains only forks of width 1 , and if $\mathbf{S}$ is the power set meetsemilattice of an arbitrary set $X$ then it contains forks of infinite width iff $X$ is infinite. In particular, the meet-semilattice of $\omega$-languages over some alphabet $\Sigma$ (see example 1) contains forks of infinite width iff $\Sigma$ is not unary. The same holds for the meet-semilattice of prefix-closed $*$-languages over $\Sigma$. This is so because if $\Sigma$ is unary then the prefix-closed $*$-languages form a chain. And if $\Sigma$ contains the distinct letters $a$ and $b$, then $Y=\left\{a^{*}\right\} \cup\left\{a^{*} \cup a^{i} b^{*} \mid i \in \mathbb{N}\right\}$ is a fork of infinite width.

In order to prove our main theorem, we need two lemmas. Lemma 3 is purely combinatorial, it states the infeasibility of particular boolean equation systems. Lemma 4 forms the core of the main theorem. Provided that the semilattice $\mathbf{S}$ contains forks of sufficient width, it reduces the existence of a sound and complete circular A-G rule $R$ which is compositional in the side condition to feasibility of a boolean equation system, which is known to be be infeasible by Lemma 3. This contradiction implies that $R$ must be unsound or incomplete.

Lemma 3. Let $m, n \in \mathbb{N}$ and $F:\{0,1\}^{m+n} \rightarrow\{0,1\}$. Then the system of equations $E_{F}$ over the variables $u_{i}^{k}\left(0 \leq i \leq 2^{\min \{m, n\}}, 1 \leq k \leq m\right)$ and $v_{j}^{l}$ $\left(0 \leq j \leq 2^{\min \{m, n\}}, 1 \leq l \leq n\right)$ has no solutions, where $E_{F}$ is defined as

$$
\begin{gathered}
E_{F}=\left\{F\left(u_{i}^{1}, \ldots, u_{i}^{m}, v_{j}^{1}, \ldots, v_{j}^{n}\right)=1 \mid 0 \leq i, j \leq 2^{\min \{m, n\}}, i \neq j\right\} \\
\cup\left\{F\left(u_{i}^{1}, \ldots, u_{i}^{m}, v_{i}^{1}, \ldots, v_{i}^{n}\right)=0 \mid 1 \leq i \leq 2^{\min \{m, n\}}\right\} .
\end{gathered}
$$


Lemma 4. Let $R: \Phi / \psi$ if $C\left[s_{1}, s_{2}, p_{1}, \ldots, p_{n}\right]$ be a circular $A-G$ rule. Let $r_{1}, r_{2} \in \mathbb{N}$, let $F:\{0,1\}^{r_{1}+r_{2}} \rightarrow\{0,1\}$ be a $\left(r_{1}+r_{2}\right)$-ary boolean function and let $C_{1}^{1}, \ldots, C_{1}^{r_{1}}, C_{2}^{1}, \ldots, C_{2}^{r_{2}}: S^{n+1} \rightarrow\{0,1\}$ be $(n+1)$-ary functions such that

$$
C\left[s_{1}, s_{2}, \tilde{p}\right]=F\left[C_{1}^{1}\left[s_{1}, \tilde{p}\right], \ldots, C_{1}^{r_{1}}\left[s_{1}, \tilde{p}\right], C_{2}^{1}\left[s_{2}, \tilde{p}\right], \ldots, C_{2}^{r_{2}}\left[s_{2}, \tilde{p}\right]\right]
$$

where $\tilde{p}$ stands for $p_{1}, \ldots, p_{n}$. If $\mathbf{S}$ contains a fork of width $2^{\min \left\{r_{1}, r_{2}\right\}}$ then $R$ is unsound or incomplete.

Proof. Without loss of generality we may assume that there is $m \in\{0, \ldots, n\}$ such that for all $j \geq 1, \Phi \models t_{\psi} \sqsubseteq p_{j}$ iff $j \leq m$, where $t_{\psi}$ is the left-hand side of $\psi$. In what follows, we sketch a proof by contradiction.

Assume that $R$ is sound and complete and let $Y \subseteq S$ be a fork of width $2^{\min \left\{r_{1}, r_{2}\right\}}$, i.e., $Y=\left\{x_{0}, x_{1}, \ldots, x_{2^{r}}\right\}$ with $r=\min \left\{\bar{r}_{1}, r_{2}\right\}$ such that for all $i, j \geq 0$ with $i \neq j, x_{0}=x_{i} \wedge x_{j}$. By Lemma 3, we know that the system of equations

$$
\begin{aligned}
\left\{F\left(u_{i}^{1}, \ldots, u_{i}^{r_{1}}, v_{j}^{1}, \ldots, v_{j}^{r_{2}}\right)\right. & \left.=1 \mid 0 \leq i, j \leq 2^{\min \left\{r_{1}, r_{2}\right\}}, i \neq j\right\} \\
\cup\left\{F\left(u_{i}^{1}, \ldots, u_{i}^{r_{1}}, v_{i}^{1}, \ldots, v_{i}^{r_{2}}\right)\right. & \left.=0 \mid 1 \leq i \leq 2^{\min \left\{r_{1}, r_{2}\right\}}\right\}
\end{aligned}
$$

over the variables $u_{i}^{k}$ and $v_{j}^{l}\left(0 \leq i, j \leq 2^{\min \left\{r_{1}, r_{2}\right\}}, 1 \leq k \leq r_{1}, 1 \leq l \leq r_{2}\right)$ has no solutions. However, we will show that there is a solution to (5), namely with $C_{1}^{k}\left(x_{i}, \widetilde{1}, \widetilde{x_{0}}\right)$ resp. $C_{2}^{l}\left(x_{j}, \widetilde{1}, \widetilde{x_{0}}\right)$ as the values of $u_{i}^{k}$ resp. $v_{j}^{l}$, where $\widetilde{1}$ abbreviates the list $1, \ldots, 1$ of length $m$, and $\widetilde{x_{0}}$ abbreviates the list $x_{0}, \ldots, x_{0}$ of length $n-m$. For all $i, j \in\left\{0, \ldots, 2^{\min \left\{r_{1}, r_{2}\right\}}\right\}$, we define a valuation $\alpha_{i j}$ such that $\alpha_{i j}\left(s_{1}\right)=x_{i}$, $\alpha_{i j}\left(s_{2}\right)=x_{j}, \alpha_{i j}\left(p_{1}\right)=\cdots=\alpha_{i j}\left(p_{m}\right)=1$ and $\alpha_{i j}\left(p_{m+1}\right)=\cdots=\alpha_{i j}\left(p_{n}\right)=x_{0}$.

First, let $i, j \in\left\{0, \ldots, 2^{\min \left\{r_{1}, r_{2}\right\}}\right\}$ with $i \neq j$. Then we can show $\alpha_{i j} \models \Phi$ (by Proposition [2) and $\alpha_{i j} \models \psi$. Hence by completeness, $\alpha_{i j} \models C\left[s_{1}, s_{2}, \tilde{p}\right]$, i. e., $C\left[s_{1}, s_{2}, \tilde{p}\right]\left(\alpha_{i j}\right)=1$, which by (44) expands to the equation

$$
F\left(C_{1}^{1}\left(x_{i}, \widetilde{1}, \widetilde{x_{0}}\right), \ldots, C_{1}^{r_{1}}\left(x_{i}, \widetilde{1}, \widetilde{x_{0}}\right), C_{2}^{1}\left(x_{j}, \widetilde{1}, \widetilde{x_{0}}\right), \ldots, C_{2}^{r_{2}}\left(x_{j}, \widetilde{1}, \widetilde{x_{0}}\right)\right)=1 .
$$

Second, let $i \in\left\{1, \ldots, 2^{\min \left\{r_{1}, r_{2}\right\}}\right\}$. Then we can show $\alpha_{i i}=\Phi$ (by Proposition 2) and $\alpha_{i i} \not \models \psi$ (using Proposition[1). Thus, soundness forces $\alpha_{i i} \not \models C\left[s_{1}, s_{2}, \tilde{p}\right]$, i. e., $C\left[s_{1}, s_{2}, \tilde{p}\right]\left(\alpha_{i i}\right)=0$, which by (44) expands to the equation

$$
F\left(C_{1}^{1}\left(x_{i}, \widetilde{1}, \widetilde{x_{0}}\right), \ldots, C_{1}^{r_{1}}\left(x_{i}, \widetilde{1}, \widetilde{x_{0}}\right), C_{2}^{1}\left(x_{i}, \widetilde{1}, \widetilde{x_{0}}\right), \ldots, C_{2}^{r_{2}}\left(x_{i}, \widetilde{1}, \widetilde{x_{0}}\right)\right)=0 .
$$

Thus, the system of equations (5) indeed has a solution, which ends this proof by contradiction.

We have shown that an A-G rule which is compositional in the side condition cannot be both sound and complete - provided that the semilattice $\mathbf{S}$ contains forks of sufficient width. The latter is the case trivially whenever $\mathbf{S}$ contains a fork of infinite width.

Theorem 5. If $\mathbf{S}$ contains forks of infinite width then there exists no sound and complete compositional circular assume-guarantee rule.

Proof. Follows from Lemma 4 . 


\section{Discussion of Related Work}

Incompleteness of Other Rules. Our setting of inference rules in meetsemilattices is a very abstract one. Most circular A-G rules in the literature are presented in more concrete settings, i. e., they use more structure than just meet and order - and that extra structure is usually indispensable for proving soundness by a circularity-breaking induction. This raises the question to what extent our incompleteness result is relevant for such concrete rules.

We claim that most circular A-G rules can be transformed - preserving soundness and compositionality - into equivalent circular A-G rules in meetsemilattices which contain forks of infinite width. Incompleteness of the transformed rule then points out a defect of the original rule: There must be cases in which the original rule is not applicable although soundness is not in danger. Below, we will exemplify two such transformations.

Various circular A-G rules have been proposed for settings, where systems and properties are presented as some form of transition graphs enriched with input and output, e. g., Moore or Mealy machines [119] or Reactive Modules 44. These rules establish certain refinement relations, e.g., trace containment or simulation, between compositions of transition graphs. Thereby, composition is a partial operation, which is defined only if the components satisfy some condition called compatibility. These compatibilities form an implicit side condition to the A-G rules, which is made explicit by the transformation. We demonstrate this in the following example by means of the transformation of a circular A-G rule for Moore machines.

Example 6. Let $\mathcal{X}$ be a finite set of variables, ranging over an arbitrary nonempty domain $\mathcal{D}$. A Moore machine $M$ is a (possibly infinite) state transition graph with input variables $I_{M} \subseteq \mathcal{X}$ and output variables $O_{M} \subseteq \mathcal{X}$, where the nodes resp. edges of the graph are labeled by valuations of the output resp. input variables; for a formal definition see for instance [9]1]. Naturally, one associates a trace language $\llbracket M \rrbracket \subseteq \Sigma^{*}$ with $M$, where $\Sigma=\mathcal{D}^{\mathcal{X}}$ is the set of valuations of all variables. The parallel composition $M_{1} \| M_{2}$ of two Moore machines $M_{1}$ and $M_{2}$ corresponds to language intersection, i. e., $\llbracket M_{1} \| M_{2} \rrbracket=\llbracket M_{1} \rrbracket \cap \llbracket M_{2} \rrbracket$. Note that $M_{1} \| M_{2}$ is defined only if $M_{1}$ and $M_{2}$ are compatible, i. e., $O_{M_{1}}$ and $O_{M_{2}}$ are disjoint.

For Moore machines with trace semantics, the following circular proof rule is known:

$$
\frac{\llbracket P_{2}\left\|S_{1} \rrbracket \subseteq \llbracket P_{1} \rrbracket \quad \llbracket P_{1}\right\| S_{2} \rrbracket \subseteq \llbracket P_{2} \rrbracket}{\llbracket S_{1}\left\|S_{2} \rrbracket \subseteq \llbracket P_{1}\right\| P_{2} \rrbracket}
$$

where $S_{1}, S_{2}, P_{1}, P_{2}$ are Moore machines such that all parallel compositions in (6) are defined. We transform this rule into the A-G rule $R_{\text {Moore }}$ for the meet-semilattice $\left\langle\mathcal{P}\left(\Sigma^{*}\right), \cap, \Sigma^{*}, \subseteq\right\rangle$, the power set of $\Sigma^{*}$ :

$$
R_{\text {Moore }}: \frac{p_{2} \sqcap s_{1} \sqsubseteq p_{1} \quad p_{1} \sqcap s_{2} \sqsubseteq p_{2}}{s_{1} \sqcap s_{2} \sqsubseteq p_{1} \sqcap p_{2}} \text { if } F\left[C\left[s_{1}\right], C\left[p_{1}\right], C\left[s_{2}\right], C\left[p_{2}\right]\right]
$$


where $D=\mathcal{P}(\mathcal{X}) \uplus\{\perp\}$ is a finite set and $C: \mathcal{P}\left(\Sigma^{*}\right) \rightarrow D$ is a finitary abstraction mapping each $L \in \mathcal{P}\left(\Sigma^{*}\right)$ to the least set of output variables $O_{M}$ such that $M$ is a Moore machine with $\llbracket M \rrbracket=L$; if no such Moore machine exists then $C(L)=\perp$. The function $F: D^{4} \rightarrow\{0,1\}$ is defined by $F\left(O_{s_{1}}, O_{p_{1}}, O_{s_{2}}, O_{p_{2}}\right)=1$ iff

$$
\begin{gathered}
O_{s_{1}} \neq \perp \text { and } O_{p_{1}} \neq \perp \text { and } O_{s_{2}} \neq \perp \text { and } O_{p_{2}} \neq \perp \\
\text { and } O_{s_{1}} \cap O_{s_{2}}=O_{p_{1}} \cap O_{p_{2}}=O_{p_{2}} \cap O_{s_{1}}=O_{p_{1}} \cap O_{s_{2}}=\emptyset \text {. }
\end{gathered}
$$

$R_{\text {Moore }}$ is a compositional circular A-G rule according to Section 3. Circularity and compositionality in the premises are obvious. Compositionality in the side condition holds as obviously there exist functions $C_{i}^{k}: \mathcal{P}\left(\Sigma^{*}\right)^{3} \rightarrow D$ such that

$$
\begin{aligned}
F\left[C\left[s_{1}\right], C\left[p_{1}\right],\right. & \left.C\left[s_{2}\right], C\left[p_{2}\right]\right] \\
& =F\left[C_{1}^{1}\left[s_{1}, p_{1}, p_{2}\right], C_{1}^{2}\left[s_{1}, p_{1}, p_{2}\right], C_{2}^{1}\left[s_{2}, p_{1}, p_{2}\right], C_{2}^{2}\left[s_{2}, p_{1}, p_{2}\right]\right] .
\end{aligned}
$$

Moreover, soundness of $R_{\text {Moore }}$ can be reduced to soundness of the original rule (6), and vice versa, so both rules are applicable in exactly the same cases. To see how soundness of $R_{\text {Moore }}$ reduces to (6), consider the premises and side condition of $R_{\text {Moore }}$ to be true under a valuation $\alpha$. Then there are Moore machines $S_{i}$ and $P_{j}$ such that $\llbracket S_{i} \rrbracket=\alpha\left(s_{i}\right)$ and $\llbracket P_{j} \rrbracket=\alpha\left(p_{j}\right)$ and $S_{1}$ and $S_{2}$, $P_{1}$ and $P_{2}, P_{2}$ and $S_{1}$ as well as $P_{1}$ and $S_{2}$ are compatible, i.e., all parallel compositions in (6) are defined. Furthermore, we have $\llbracket P_{2} \rrbracket \cap \llbracket S_{1} \rrbracket \subseteq \llbracket P_{1} \rrbracket$ and $\llbracket P_{1} \rrbracket \cap \llbracket S_{2} \rrbracket \subseteq \llbracket P_{2} \rrbracket$, which by language intersection and soundness of (66) implies $\llbracket S_{1}\left\|S_{2} \rrbracket \subseteq \llbracket P_{1}\right\| P_{2} \rrbracket$, which in turn by language intersection implies that the conclusion of $R_{\text {Moore }}$ is true under $\alpha$. To show the converse reduction, let $S_{i}$ and $P_{j}$ be Moore machines such that all parallel compositions in (6) are defined, i. e., $S_{1}$ and $S_{2}, P_{1}$ and $P_{2}, P_{2}$ and $S_{1}$ as well as $P_{1}$ and $S_{2}$ are compatible. Obviously, soundness of (6) follows by language intersection and soundness of $R_{\text {Moore }}$.

As the proof rule (6) has been proven sound in [11, $R_{\text {Moore }}$ is a sound and compositional circular A-G rule. Thus by Theorem 5, $R_{\text {Moore }}$ is incomplete because the meet-semilattice $\left\langle\mathcal{P}\left(\Sigma^{*}\right), \cap, \Sigma^{*}, \subseteq\right\rangle$ contains forks of infinite width. Hence there are cases in which circular reasoning is admissible yet the rule (6) is not applicable, due to partiality of parallel composition.

Other kinds of circular A-G rules focus on temporal logics to present properties (and sometimes systems also), see for instance [1210]. In order to break the circularity, such rules usually employ so-called assume-guarantee specifications, i. e., formulas of the form $\varphi \triangleright \psi$ where $\triangleright$ is a special temporal operator ensuring that during any computation the guarantee $\psi$ holds at least one step longer than the assumption $\varphi$. In our meet-semilattice setting, we cannot express A-G specifications in the premises of inference rules. However, we can move A-G specifications to the side condition, where their truth is expressible as a relation. In the following example, we demonstrate this transformation on a simple circular rule for A-G specifications.

Example 7. Let $A P$ be a non-empty set of atomic propositions. We say that $\Sigma=\mathcal{P}(A P)$ is the set of states, and $\Sigma^{\omega}$ is the set of computations. A system 
is a set of computations, and the parallel composition of two systems $S_{1}$ and $S_{2}$ is their intersection $S_{1} \cap S_{2}$. Likewise, a property is a set of computations, and we say that a property $P$ entails another property $Q$ iff $P \subseteq Q$. We may represent certain properties by formulas in linear-time temporal logic (LTL), which are constructed from atomic propositions by means of boolean operators and the standard temporal operators X (next-time), U (until), F (eventually) and $\mathrm{G}$ (always); for a formal definition of syntax and semantics of LTL see for instance [5. Henceforth, we will identify a formula $\varphi$ with the property it represents.

Given two formulas $\varphi$ and $\psi$, we define the assume-guarantee specification $\varphi \triangleright \psi$ as an abbreviation of the formula $\neg(\varphi \mathrm{U} \neg \psi)$, cf. [18]. The temporal operator $\triangleright$ satisfies the following (in)equalities:

$$
\begin{gathered}
\varphi \triangleright \psi=\psi \wedge(\varphi \Rightarrow \mathrm{X}(\varphi \triangleright \psi)) \\
\mathrm{G} \varphi \wedge(\varphi \triangleright \psi) \subseteq \mathrm{G} \psi
\end{gathered}
$$

From the fix-point equation (77), we can read off that $\varphi \triangleright \psi$ is the weakest property where $\psi$ holds strictly longer than $\varphi$ along every computation.

For A-G specifications, the following circular proof rule is known:

$$
\frac{S_{1} \subseteq \varphi_{2} \triangleright \varphi_{1} \quad S_{2} \subseteq \varphi_{1} \triangleright \varphi_{2}}{S_{1} \cap S_{2} \subseteq \mathrm{G}\left(\varphi_{1} \wedge \varphi_{2}\right)}
$$

where $S_{1}, S_{2}$ are systems and $\varphi_{1}, \varphi_{2}$ are LTL formulas. We transform this rule into the A-G rule $R_{\triangleright}$ for the meet-semilattice of systems and properties $\left\langle\mathcal{P}\left(\Sigma^{\omega}\right), \cap, \Sigma^{\omega}, \subseteq\right\rangle:$

$$
R_{\triangleright}: \frac{p_{4} \sqcap s_{1} \sqsubseteq p_{3} \quad p_{3} \sqcap s_{2} \sqsubseteq p_{4}}{s_{1} \sqcap s_{2} \sqsubseteq p_{3} \sqcap p_{4}} \text { if } C_{1}^{1}\left[s_{1}, p_{1}, \ldots, p_{4}\right] * C_{2}^{1}\left[s_{2}, p_{1}, \ldots, p_{4}\right]
$$

where $*:\{0,1\}^{2} \rightarrow\{0,1\}$ denotes multiplication (i.e., conjunction in logical terms) and the functions $C_{i}^{k}: \mathcal{P}\left(\Sigma^{\omega}\right)^{5} \rightarrow\{0,1\}$ are defined by

$$
\begin{aligned}
& C_{1}^{1}\left(S_{1}, P_{1}, P_{2}, P_{3}, P_{4}\right)=1 \text { iff } P_{3}=\mathrm{G} P_{1} \text { and } P_{4}=\mathrm{G} P_{2} \text { and } S_{1} \subseteq P_{2} \triangleright P_{1}, \\
& C_{2}^{1}\left(S_{2}, P_{1}, P_{2}, P_{3}, P_{4}\right)=1 \text { iff } P_{3}=\mathrm{G} P_{1} \text { and } P_{4}=\mathrm{G} P_{2} \text { and } S_{2} \subseteq P_{1} \triangleright P_{2} .
\end{aligned}
$$

Note that the equality $P_{3}=\mathrm{G} P_{1}$ is supposed to hold iff there exists an LTL formula $\varphi_{1}$ such that $\varphi_{1}$ and $G \varphi_{1}$ represent the properties $P_{1}$ and $P_{3}$, respectively; $P_{4}=\mathrm{G} P_{2}$ is to be interpreted similarly.

Obviously, $R_{\triangleright}$ is a compositional circular A-G rule1 according to Section 3 Moreover, soundness of $R_{\triangleright}$ can be reduced to soundness of the original rule (9), and vice versa, so both rules are applicable in exactly the same cases. To see how soundness of $R_{\triangleright}$ reduces to (9), consider the premises and side condition of $R_{\triangleright}$ to be true under a valuation $\alpha$. Then there are LTL formulas $\varphi_{j}$ such that $\mathrm{G} \varphi_{1}=\alpha\left(p_{3}\right)$ and $\mathrm{G} \varphi_{2}=\alpha\left(p_{4}\right)$ and $\alpha\left(s_{1}\right) \subseteq \varphi_{2} \triangleright \varphi_{1}$ and $\alpha\left(s_{2}\right) \subseteq \varphi_{1} \triangleright \varphi_{2}$. Using soundness of (9), we infer $\alpha\left(s_{1}\right) \cap \alpha\left(s_{2}\right) \subseteq \mathrm{G}\left(\varphi_{1} \wedge \varphi_{2}\right)$, which implies that the

\footnotetext{
${ }^{1}$ The trivial premises $p_{1} \sqsubseteq \top$ and $p_{2} \sqsubseteq \top$ have been omitted from the definition of $R_{\triangleright}$ for the sake of readability.
} 
conclusion of $R_{\triangleright}$ is true under $\alpha$. To show the converse reduction, let $S_{i}$ and $\varphi_{j}$ be systems and formulas, respectively, such that the premises of rule (9) hold. By (8), these premises imply $\mathrm{G} \varphi_{2} \cap S_{1} \subseteq \mathrm{G} \varphi_{1}$ and $\mathrm{G} \varphi_{1} \cap S_{2} \subseteq \mathrm{G} \varphi_{2}$, respectively. Using soundness of $R_{\triangleright}$, we infer $S_{1} \cap S_{2} \subseteq \mathrm{G} \varphi_{1} \cap \mathrm{G} \varphi_{2}$, which is equivalent to the conclusion of (9).

As the proof rule (9) is sound, cf. [16 18], $R_{\triangleright}$ is a sound and compositional circular A-G rule. Thus by Theorem $5 R_{\triangleright}$ is incomplete because the meetsemilattice $\left\langle\mathcal{P}\left(\Sigma^{\omega}\right), \cap, \Sigma^{\omega}, \subseteq\right\rangle$ contains forks of infinite width. As a consequence, the rule (9) does not capture all sound circular reasoning patterns, i.e., there are cases in which circular reasoning is admissible yet (9) is not applicable.

In short, this paper shows that compositionality implies incompleteness. Yet, we did not encounter any complete rule except for the rather trivial rule $R_{2}$ from example 2. This raises the question whether non-trivial sound and complete circular A-G rules do exist at all. They do - in [12, we present a very general sound and complete circular A-G rule for certain classes of lattices. Of course, that rule must be non-compositional; in fact, it is non-compositional both in the premises and in the side condition. Still, that general rule can be instantiated to many known circular A-G rules, no matter whether they are compositional or not.

Other Notions of Completeness. When some complex system should be verified against a conjunction of properties, one usually applies backward reasoning, i. e., one matches the verification goal against the conclusion of a proof rule and from the premises and the side condition one infers the subgoals that need to be established. In [18], the authors investigate a notion of completeness that characterizes rules which always enable backward reasoning, so we will term this notion backward completeness. Adopted to our setting, a rule $R: \Phi / \psi$ if $\Gamma$ is called backward complete iff for all valuations $\alpha, \alpha \models \psi$ implies $\alpha^{\prime} \models \Phi$ and $\alpha^{\prime} \models \Gamma$ for some valuation $\alpha^{\prime}$ which agrees with $\alpha$ on the variables of $\psi$. Thus, truth of the conclusion implies that the premises and the side condition can be made true through choosing (i. e., guessing) appropriate values for the auxiliary variables, i. e., for those variables in the premises that do not occur in the conclusion. Note that backward completeness does not distinguish premises and side condition, whereas this distinction is essential for our notion of completeness.

Our notion of completeness relates more to forward reasoning, i. e., from prior knowledge which subsystems guarantee which properties assuming which other properties, we want to infer that the complex system guarantees a conjunction of properties. A complete rule (in the sense of this paper) will enable this inference whenever the conclusion is consistent with our knowledge. Still, our incompleteness result bears some significance for backward complete rules. For a rule $R: \Phi / \psi$ if $\Gamma$ without auxiliary variables, i. e., $\operatorname{var}(\psi)=\operatorname{var}(\Phi)$, backward completeness implies completeness. Thus, as a consequence of Theorem 5 every sound and backward complete compositional circular A-G rule necessarily needs to employ auxiliary variables. In other words, backward reasoning with compositional circular rules is likely to require guessing auxiliary assertions about the 
system. I. e., one trades the lower complexity of the (decomposed) system for a higher complexity of the proof search.

\section{Conclusion}

We have shown that sound and compositional circular assume-guarantee rules, presented as inference rules restricted by an arbitrary side condition, cannot be complete. I. e., the side condition of a compositional rule, no matter how elaborate it is, cannot capture all cases where circular reasoning is admissible. Consequently, two important criteria for rating the quality of inference rules work against each other in the realm of circular reasoning. Upon designing assumeguarantee rules, this raises the question whether we should settle for compositionality or rather for completeness. The answer depends on the intended use of the rule.

Over the years, the practicality of circular assume-guarantee reasoning as a technique for compositional verification has been documented in a number of case studies, see [7/815] to name a few. In most cases, these assume-guarantee rules were tailored for model checking, and as model checkers particularly suffer from the infamous state explosion problem, the designers of the rules focussed on (automatic) system decomposition rather than on completeness. Consequently, these rules avoid to generate subgoals that involve a composition of subsystems. Here, compositional rules whose side conditions can be checked efficiently (but are not too restrictive) seem to be very appropriate. There are tools that successfully employ such incomplete compositional rules, e.g., in the verification of thread-parallel software [6]. To some extent, the loss of completeness can be mitigated against by human interaction, e.g., in the form of auxiliary annotations (to the code of the system), which provide more information about the system so the tools may find better decompositions.

To the best of our knowledge, there is no data available on the practical use of complete circular assume-guarantee rules in verification. However, in the case of manual (or almost manual) verification, we see no reason for severely restricting the power of circular reasoning, so one might prefer a complete rule over a compositional one. Of course, then one must tackle system decomposition in the subgoals by other means, e.g., by abstraction. Still, assume-guarantee reasoning may be superior to direct verification, as the additional assumptions in the subgoals may enable better abstractions.

Acknowledgement. The author thanks Viorica Sofronie-Stokkermans and Andreas Podelski for helpful discussions and comments and Carsten Sinz for support with theorem provers.

\section{References}

1. M. Abadi and L. Lamport. Conjoining specifications. ACM Transactions on Programming Languages and Systems, 17(3):507-534, 1995. 
2. M. Abadi and S. Merz. An abstract account of composition. In MFCS, LNCS 969, pages 499-508. Springer, 1995.

3. B. Alpern and F. B. Schneider. Defining liveness. Information Processing Letters, 21:181-185, 1985.

4. R. Alur and T. A. Henzinger. Reactive modules. In LICS, pages 207-218. IEEE Computer Society, 1996.

5. E. A. Emerson. Modal and temporal logics. In J. van Leeuwen, editor, Handbook of Theoretical Computer Science, volume B, pages 135-191. Elsevier, 1990.

6. C. Flangan, S. N. Freund, and S. Qadeer. Thread-modular verification for sharedmemory programs. In ESOP, LNCS 2305, pages 262-277. Springer, 2002.

7. T. A. Henzinger, X. Liu, S. Qadeer, and S. K. Rajamani. Formal specification and verification of a dataflow processor array. In ICCAD, pages 494-499. IEEE Computer Society, 1999.

8. T. A. Henzinger, S. Qadeer, and S. K. Rajamani. You assume, we guarantee: Methodology and case studies. In $C A V$, LNCS 1427, pages 440-451. Springer, 1998.

9. T. A. Henzinger, S. Qadeer, S. K. Rajamani, and S. Tasiran. An assume-guarantee rule for checking simulation. ACM Transactions on Programming Languages and Systems, 24(1):51-64, 2002.

10. B. Jonsson and Y.-K. Tsay. Assumption/guarantee specifications in linear-time temporal logic. Theoretical Computer Science, 167(1-2):47-72, 1996.

11. P. Maier. A set-theoretic framework for assume-guarantee reasoning. In ICALP, LNCS 2076, pages 821-834. Springer, 2001.

12. P. Maier. A Lattice-Theoretic Framework For Circular Assume-Guarantee Reasoning. PhD thesis, Universität des Saarlandes, 2002. Submitted.

13. P. Maier. Compositional circular assume-guarantee rules cannot be sound and complete. Technical Report MPI-I-2003-2-001, Max-Planck-Institut für Informatik, 2003.

14. Z. Manna and A. Pnueli. Temporal Verification of Reactive Systems: Safety. Springer, 1995.

15. K. L. McMillan. Verification of an implementation of Tomasulo's algorithm by compositional model checking. In $C A V$, LNCS 1427, pages 110-121. Springer, 1998.

16. K. L. McMillan. Circular compositional reasoning about liveness. In CHARME, LNCS 1703, pages 342-345. Springer, 1999.

17. J. Misra and K. M. Chandy. Proofs of networks of processes. IEEE Transactions on Software Engineering, 7(4):417-426, 1981.

18. K. S. Namjoshi and R. J. Trefler. On the completeness of compositional reasoning. In $C A V$, LNCS 1855, pages 139-153. Springer, 2000.

19. W. Thomas. Automata on infinite objects. In J. van Leeuwen, editor, Handbook of Theoretical Computer Science, volume B, pages 135-191. Elsevier, 1990.

20. M. Viswanathan and R. Viswanathan. Foundations for circular compositional reasoning. In ICALP, LNCS 2076, pages 835-847. Springer, 2001. 